\title{
Research Paper: Distress Tolerance, Impulsivity, and Aggression: The Role of Emotional Dysregulation and Reward Sensitivity in Individuals With Borderline Personality Disorder Features
}

\author{
Zahra Shadara $^{1^{*}}$ (D), Mohsen Dehghani ${ }^{1}$ (D), Mahmoud Heidari ${ }^{1}$ (D), Majid Mahmoud Aliloo² ${ }^{\text {(D) }}$ \\ 1. Department of Clinical Psychology, Faculty of Educational Science and Psychology, Shahid Beheshti University, Tehran, Iran. \\ 2. Department of Clinical Psychology, Faculty of Educational Science and Psychology, Tabriz University, Tabriz, Iran.
}

\begin{tabular}{|c|c|}
\hline & \\
\hline arestip & $\begin{array}{l}\text { sion: The Role of Emotional Dysregulation and Reward Sensitivity in Individuals With Borderline Personality Disorder Features. } \\
\text { Journal of Practice in Clinical Psychology, 9(1), 37-50. https://doi.org/10.32598/jpcp.9.1.727.1 }\end{array}$ \\
\hline atives & dol'https://doi.org/10.32598/jpcp.9.1.727.1 \\
\hline
\end{tabular}

(c) (i) (5)

Article info:

Received: 20 Sep 2020

Accepted: 10 Dec 2020

Available Online: 01 Jan 202

Keywords:

Borderline personality disorders, Emotional regulations, Delayed gratification, Emotional distress, Impulsivity, Aggression

\section{ABSTRACT}

Objective: Borderline Personality Disorder (BPD) is one of the most severe psychiatric disorders. This study aimed to predict distress tolerance, impulsivity, and aggression based on emotional dysregulation and reward sensitivity among individuals with BPD features.

Methods: The study population was undergraduate students of Shahid Beheshti University, Tehran City, Iran, in the academic year 2017-18. A total of 1005 male and female students were chosen based on the available sampling method and were screened according to the personality assessment inventory-borderline scale. Finally, 146 subjects (50 males and 96 females) could reach the second phase of the research, and all of them completed the difficulties in emotion regulation scale, distress tolerance scale, Balloon analog risk task as well as aggression questionnaire. To analyze the data, we used multivariate regression analysis.

Results: Results of the current research showed that emotional dysregulation and its components had a predictive role in distress tolerance and aggression. On the other hand, only reward sensitivity could have an essential role in predicting impulsivity in individuals with BPD features.

Conclusion: This study reflects the remarkable role of emotional regulation mechanisms in distress tolerance and aggression, and in contrast, the role of biological mechanisms such as sensitivity to reward in impulsivity among individuals with BPD features. Furthermore, the present research results can have clinical implications considering both etiology and treatment of BPD patients.

\section{* Corresponding Author:}

Zahra Shadara, MSc.

Address: Department of Clinical Psychology, Faculty of Educational Science and Psychology, Shahid Beheshti University, Tehran, Iran.

Tel: +98 (21) 29905305

E-mail: zahrash549@gmail.com 


\section{Highlights}

- Emotional dysregulation and its all components played a pivotal role in predicting distress tolerance as well as aggression.

- Reward sensitivity as a biological mechanism showed a remarkable effect in impulsivity among individuals with Borderline personality disorder features.

\section{Plain Language Summary}

The results of this study showed the importance of emotional regulation in diagnostic symptoms such as aggression and distress tolerance in comparison to the role of biological factors in predicting impulsivity. Therefore, we can consider clinical implications according to the current findings.

\section{Introduction}

orderline Personality Disorder (BPD) is one of the most severe psychiatric conditions, with a high prevalence of between $1 \%$ and $3 \%$ in the general population (Trull, Jahng, Tomko, Wood, \& Sher, 2010). According to the DSM-5 diagnostic criteria, the impairments of interpersonal functioning, affective dysregulation, and impulsivity are major symptom clusters in this disorder (American Psychiatric Association, 2013). Additionally, BPD patients suffer from low distress tolerance when exposed to environmental stressors (Bornovalova, Matusiewicz, \& Rojas, 2011). Distress tolerance refers to individuals' capacity to resist unpleasant mental states (Leyro, Zvolensky, \& Bernstein, 2010), which is reported to be remarkably low in BPD patients. Furthermore, conducting harmful behaviors such as impulsivity is considered a consequence of low distress tolerance among BPD patients. In this regard, impulsivity has also been suggested as an essential clinical symptom in this disorder (Paris, Gunderson, \& Weinberg, 2007), which includes actions that are dangerous and disproportionate to the situation, performed carelessly, and usually with negative consequences (Evenden, 1999).

High-risk driving, drug and alcohol abuse, unprotected sexual relations, overeating, and gambling are some of the impulsive behaviors in BPD patients (American Psychiatric Association, 2013). On the other hand, impulsivity can also trigger aggressive actions in these individuals (Goodman \& New, 2000). Aggression against oneself (non-suicidal self-harm behaviors or suicide attempts) and aggression against others (outspokenness, intense conflict, throwing objects at others) are other major symptoms of this disorder (Sansone \& Sansone,
2012), which create many problems for patients and those around them. Therefore, identifying psychological and biological mechanisms that underlie distress tolerance, impulsivity, and aggression is necessary for improving treatment in BPD patients.

In this regard, emotional dysregulation is proposed as a psycho-biological mechanism. Linehan (1993) considers it as frequent fluctuations in emotions, extreme emotional responsiveness to events, and intense emotional experience. Several studies have focused on the role of emotional dysregulation in aggressive behavior (Scott, Stepp, \& Pilkonis, 2014; Martino et al., 2015), impulsivity (Baer et al., 2018; Sebastian et al., 2014; Chapman, Leung, \& Lynch, 2008), and psychological distress (Dixon-Gordon, Turner, Rosenthal, \& Chapman, 2017; Ebner-Priemer et al., 2008) in BPD patients and psychiatric disorders (Jakubczyk et al., 2018; Muratori, Pisano, Milone, \& Masi, 2017).

However, there are conflicting studies in the field of emotional regulation strategies in BPD patients that should be addressed. Research suggests that some adaptive coping strategies may exacerbate negative emotional states and physiological arousal. Additionally, it increases emotional distress in these patients. The results of the Dixon-Gordon study (Dixon-Gordon et al., 2017) showed that BPD patients in suppression state versus emotional acceptance showed decreased emotional anger, decreased postural anger, and decreased heart rate. It has also been suggested that the suppression strategy in reducing negative emotions is more effective than the acceptance strategy and reduces irritating thoughts related to stimuli (Evans, Howard, Dudas, Denman, \& Dunn, 2013). In this case, a study (Kuo, Fitzpatrick, Krantz, \& Zeifman, 2018) showed that high-intensity BPD patients reported increased positive emotions and decreased internal urge to engage in dysfunctional behaviors following emotional suppression 
rather than emotional observation. In contrast, low-intensity BPD patients showed more negative emotions and risk-taking behaviors following a suppression state.

Apart from psychological factors, biological mechanisms have recently found a salient place in the psychopathology of mental disorders. Dysfunctional responses to reward are proposed to underlie and persist in abnormality (Vollum et al., 2007). Hence, Reward Sensitivity (RS) is considered a biological factor, which refers to seek conditional and unconditional rewarding stimuli (Dawe, Gullo, \& Loxton, 2004). Additionally, extreme values of sensitivity to reward can be pointed out as a risk factor for psychological disorders (Berenson et al., 2020; Bijtebier et al., 2009). Research on the general population (Simons, Sistad, Simons, \& Hansen, 2018; Allen, \& Gabbay, 2013), patients diagnosed with major depressive disorder (Buckner, Keough, \& Schmidt, 2007), and substance abuse disorder (Bornovalova, Gratz, Daughters, Hunt, \& Lejuez, 2012) has shown that emotional distress is associated with a higher risk of appetitive behaviors. Rewarding behaviors to reduce negative emotions seem to be an indicator of people who have low distress tolerance. This group uses maladaptive strategies that quickly reduce negative emotionality (Simons \& Gaher, 2005). On the other hand, research that can show the relationship between distress tolerance and rewarding behaviors in BPD patients has not been performed and needs to be investigated.

In the context of BPD, the impulsivity of these patients, which is characterized by behavioral disinhibition, choosing instant pleasures, and the tendency to devalue long-term rewards, is associated with reward sensitivity (Lawrence, Allen, \& Chanen, 2010; KrauseUtz et al., 2016; Volker et al., 2009). Results of a metaanalysis study by using temporal delay discounting task, reversal learning, and Iowa gambling task showed that BPD patients experience altered subjective valuation of outcomes in the context of appetitive stimuli, prefer immediate rewards to delayed ones, and frequently make harmful decisions (Paret, Jennen-Steinmetz, \& Schmahl, 2016). Additionally, research on several psychiatric disorders (Curry et al., 2018; Martino et al., 2015) shows a link between reward-seeking and impulsive behaviors. On the contrary, a study (Lawrence et al., 2010) has been demonstrated that although BPD patients show devaluation of delayed rewards compared with healthy controls, they are no different from the healthy group when responding to rewarding stimuli.

Studies among adolescents and students have also shown that aggression or being in an angry state are associated with a tendency to be engaged in rewarding behaviors and ap- proach motivation (Bossuyt, Moors, \& De Houwer, 2014; Drnas, 2020; Zhao, Kirwen, Johnson, \& Vigo, 2017). Preferring instant rewards to delayed choices, approach motivation towards anger-related stimuli, and venting internal anger by aggressive attempts are usually observed in individuals with high sensitivity to reward in angry conditions. However, examining this relationship in BPD patients is a research gap that the present study addresses.

According to the consistent and inconsistent studies in the research literature, as well as the lack of integrated research that can simultaneously examine psychological and biological mechanisms involved in BPD patients, the present study intends to clarify these mechanisms. It identifies which factor plays a prominent role in this group. Moreover, the current study predicts distress tolerance, impulsivity, and aggression based on emotional dysregulation and reward sensitivity in individuals with BPD features.

\section{Methods}

The method of the present study is descriptive and correlational. The statistical population of the present study was undergraduate students of Shahid Beheshti University, Tehran City, Iran, in the academic year 2017-2018. A total of 1005 male and female students were chosen by an available sampling method. In the second phase of the research, 180 subjects with a score equal to 37 or above on the Personality Assessment Inventory-Borderline subscale (PAI-BOR) were chosen by purposive sampling method. The sample size was calculated by $\mathrm{G} \times$ Power software in correlational studies. Thirty subjects were excluded from the study due to the lack of cooperation, and 4 subjects were excluded due to their incomplete questionnaires. Finally, 146 participants (50 males and 96 females) entered the second phase of the research.

The inclusion criteria consisted of being an undergraduate student at Shahid Beheshti University in 2017-2018 (except psychology or consulting students). According to the PAI-BOR, they must have a score equal to 37 or above and participate in the first and second research phases. The exclusion criteria were no use of the substance and antipsychotic medication. These items were asked in an interview conducted by a trained clinical psychologist.

\section{Study instruments}

\section{Personality Assessment Inventory-Borderline Subscale (PAI-BOR)}

This scale consists of 24 items representing four basic symptoms in BPD patients, including emotional instabil- 
ity, self-harm/impulsivity, interpersonal relationships, and identity problems. It has a cut-point of 37 and above (Morey, 1991). Some studies have pointed out the validity and reliability of this scale for being used in non-clinical samples (Trull, 1995). This scale has been translated in Iran, and its validity and reliability have been estimated by Esmaeilian, Dehghani, Koster and Hoorelbeke (2019). In this case, after translating the questionnaire, its content validity was evaluated by experts in this field using Content Validity Index (CVI) and Content Validity Ratio (CVR). The results showed that the questions utilized in the questionnaire are useful, clear, and relevant. Besides, the convergent and divergent validity of the questionnaire and the fit indices in AMOS software were examined. The results showed that Combined Reliability (CR) was established for all subscales $(C R>0.7)$. Necessary and sufficient conditions for construct validity were also established. This scale also indicates the desired internal consistency (the Cronbach alpha=0.75). In the present study, The Cronbach alpha value was 0.84 .

\section{Difficulties in Emotion Regulation Scale (DERS)}

Difficulties in Emotion Regulation Scale (DERS) is a 36-item self-reported scale that examines the patterns of emotion regulation in individuals and has 6 components: non-acceptance of emotional responses, targeted behavior difficulties, impulse control difficulties, lack of emotional awareness, limited access to emotional regulation strategies, and lack of emotional clarity (Gratz, \& Roemer, 2004). Gratz and Roemer reported the internal consistency of this scale by the Cronbach alpha value of 0.93 for the whole scale and $0.85,0.89,0.86,0.80,0.88$, and 0.84 for its subscales, respectively. In Iran, Azizi, Mirzaei, and Shams (2010) have reported the Cronbach alpha value of 0.92 for this scale. In the current study, the Cronbach alpha was found 0.93 .

\section{Distress Tolerance Scale (DTS)}

The Dstress Tolerance Scale (DTS) was developed by Simons and Gaher (2005) and includes 15 items with four components of tolerance, evaluation, absorption, and adjustment. The internal consistency of this scale was estimated by the Cronbach alpha method for subscales of tolerance, evaluation, absorption, and adjustment as 0.72 , $0.82,0.78$, and 0.70 , respectively, and 0.82 for the whole scale. This questionnaire was used for the first time in Iran by Kakabraei and Azami (2017). The reliability by internal consistency method was found 0.71 for the whole subscale, 0.54 for the tolerance subscale, 0.42 for absorption, 0.56 for evaluation, and 0.58 for adjustment. In the current sample, the Cronbach alpha value was found 0.81 .

\section{Balloon Analog Risk Task (BART)}

The Balloon Analog Risk Task (BART) was first introduced in 2002 by Lejuez et al. (2002). This computer test examines the possibility of risk-taking in real situations and measures the functional and also dysfunctional risk-taking strategies in person. The test has been designed so that the subject's initial risk-taking is accompanied by reward. His uncontrolled risk-taking is accompanied by losses (profit and loss in the form of hypothetical rewards or fines).

\section{How to Perform BART}

In the BART test, a balloon is displayed on the computer monitor, next to which there are buttons that each time it is pressed, the diameter of the balloon increases by one degree (about $0.3 \mathrm{~cm}$ ). Each time the balloon is inflated, 5000 tomans of money is added to the reserve of a temporary fund. When the balloon inflates more than a certain amount, it pops and disappears with a "pop" sound, and the money stored in the temporary box is lost. Instead of inflating more balloons at each stage of the test, the participant can transfer the saved money to the main box by pressing another button shown on the screen. The next uninflated balloon will be given to him/ her (The total number of balloons is 30 ). The probability of a balloon exploding is based on a random selection from a sequence of numbers from 1 to 128 (selecting any number is equivalent to bursting a balloon). So that if the number 1 is chosen randomly for the first inflation of the balloon (probably 1.128), the balloon will explode at the same stage. But if another number is selected from the set of numbers 2 to 128 , that number will be removed from the set of numbers.

The balloon does not burst, but the probability of random selection of the number 1 increases to 2.128 next time. This probability will be 1 in the 128 choices. Each time the balloon is inflated, it increases the likelihood of bursting and reduces the relative amount of profit earned, so that, for example, inflating for the second time only risks 500 RLL can be a profit equal to $100 \%$. But inflating for 500 times 25000 RLL in the temporary fund endangers, and only $500 \mathrm{RLL}$, equivalent to $1.6 \%$, goes to the person. This seems to be true even in the real world of risk.

Furthermore, the test is designed to increase the amount of money received exponentially each time it blows after the 50th load. Accordingly, each time the balloon is inflated after the 50th load, instead of an increase of 500 RLL, it will be accompanied by an increase equivalent to 1000 RLL for each inflation, which is the same amount 
up to the 60 times, but from inflating 60 to 70, 1500 RLL. Increase from 70 to 80,2000 RLL, and so on until the end (every 10 more blows adds more money to the person's temporary fund). This is because as the probability of a balloon exploding at inflations above 50, more rewards are needed to motivate the person to continue to take risks. This test measures the reward sensitivity of the subject.

In BART, The Following Values Are Considered as Test Scores

1. Adjusted score: Equivalent to the average number of times an inflated balloon is pumped. This variable is the main test score and the subject's risk-taking and impulsivity index.

2. The number of balloons that have been inflated more than 50 times. This variable is considered as an indicator of reward sensitivity and reward stability.

3. The Average number of pumps in post-burst attempts. This variable is added by Nasri, Nazari, Mighani, Shahrokhi, and Goudarzi (2018) to examine punishment sensitivity in the subjects because the bursting of a balloon can be considered as a punishment. This variable has not been studied in the present study.

\section{Aggression Questionnaire}

The aggression questionnaire is a 29-item questionnaire developed by Buss and Perry (1992) and measures four aspects of aggression, physical aggression, verbal aggression, anger, and hostility. The internal consistency values of this questionnaire calculated by the Cronbach alpha method for the subscales of physical aggression, verbal aggression, anger, and hostility are $0.85,0.72,0.83,0.77$, respectively, and for the total score is 0.89 . Also, to measure its reliability by test-retest method, Buss and Perry (1992) found the correlation coefficient of 0.80 for the physical aggression subscale, 0.76 for the verbal aggression, 0.72 for the anger, 0.72 for the hostility, and 0.80 for the whole scale. Samani (2007) has reported the reliability by the test-retest method as 0.74 for anger, 0.78 for physical aggression, 0.68 for verbal aggression, and 0.68 for hostility. The Cronbach alpha values of $0.83,0.79$, 0.77 , and 0.70 were estimated for subscales. In the present study, the Cronbach alpha was found 0.84 .

\section{Study procedure}

To carry out the first phase of the research, we obtained the approval of the distribution of the questionnaire among the students from Shahid Beheshti University's
Research Ethics Committee. PAI-BOR was given to undergraduate students whose majors were not psychology and counseling (due to the potential bias). Besides, they were asked to enter their contact number to establish communication and record the research's second phase. The questionnaires were collected from 1005 subjects, and after making sure that the desired number of subjects was reached (based-on the cut-point of 37 or above), the second phase of the research began. Then, the subjects were called and given a brief explanation about their responses to the screening scale, alongside the need for the second phase of the research. Those individuals were asked to refer to the Educational Sciences and Psychology Faculty of Shahid Beheshti University. At this phase, 30 subjects were unwilling to cooperate, and therefore the study began with 150 participants. The inclusion and exclusion criteria were applied to all subjects. Finally, after the study, the questionnaires were evaluated, and 4 subjects were excluded from the study due to their incomplete questionnaires. Subsequently, scores of 146 subjects were analyzed.

This study was approved by Shahid Beheshti University Research Ethics Committee. Written consent was obtained from all participants. They were assured that their responses would remain confidential. Moreover, the participants were informed that they can get the results after the completion of the research.

Data analysis was conducted in SPSS V. 20. In descriptive statistics methods, frequency, mean, and standard deviation were reported. Multivariate regression analysis was used to assess research hypotheses.

\section{Results}

Descriptive statistics and the results of multivariate regression analysis are presented in Tables 1, 2, and 3.

According to Table 3, skewness and kurtosis values were used to examine the regression assumption based on the normality of the research variables. Then the Variance Inflation Factor (VIF) and tolerance index were applied to analyze the linear relationships between the independent variables. According to the values of skewness and kurtosis (under 1.96), VIF (under 5), and tolerance index (between 1 and 0 ), it can be put forward that regression assumptions have been observed. Afterward, multivariate regression analysis was performed by inter-method. The results are shown in Tables 4, 5, and 6, respectively.

As seen in Table 4, the multiple correlation coefficient of predictor variables (components of emotional dys- 
regulation and reward sensitivity) with distress tolerance is equal to 0.703 , and the coefficient of determination is 0.494 , which is significant according to ANOVA test results (19.274) at the significance level of $<0.0001$. This means that components of emotional dysregulation and reward sensitivity explain $49.4 \%$ vari ance of distress tolerance on the whole. Regression coefficient results also show the strongest predictors of distress tolerance were limited access to emotion regulation strategies $(\beta=$ $-0.344)$ and none-acceptance of emotional responses $(\beta=$ $-0.308)$, respectively. Other components did not play a predictive role in this regression model.

Table 5 shows the multiple correlation coefficient of predictor variables (components of emotional dysregulation and reward sensitivity) with impulsivity of 0.925 and the coefficient of determination of 0.856 , which is significant based on the F factor (117.247) at the significance level of $<0.0001$. This finding states that $85.6 \%$ variance of impulsivity can be explained by components of emotional dysregulation and reward sensitivity. On the other hand, according to regression coefficients, the only predictor of impulsivity was reward sensitivity $(\beta=0.914)$. Components of emotional dysregulation did not have an essential role in impulsivity.

According to Table 6, the multiple correlation coefficient of predictor variables with aggression is 0.397 . The coefficient of determination is equal to 0.158 , which is significant according to $\mathrm{F}$ at the significance level of $<0.001$. This result puts forward that the components of emotional dysregulation and reward sensitivity together account for $15.8 \%$ of the variance in aggression. Also, according to regression coefficients, the only predictor of aggression was impulse control difficulties. Other components did not predict aggression.

On the other hand, the MANOVA (Multivariate Analysis of Variance) method was used to analyze the effect of BPD features severity on participants' performance

Table 1. Demographic indicators of participants

\begin{tabular}{ccc}
\hline Participants & Mean \pm SD & N \\
\hline Male & $20.66 \pm 1.54$ & 50 \\
\hline Female & $20.75 \pm 1.81$ & 96 \\
Total & $20.72 \pm 1.72$ & 146 \\
\hline & & PRACTICE In \\
\hline
\end{tabular}

Table 2. Descriptive statistics of the research variables $(\mathrm{N}=146)$

\begin{tabular}{|c|c|}
\hline Variables & Mean士SD \\
\hline Emotional dysregulation & $108.93 \pm 20.66$ \\
\hline Non-acceptance of emotional responses & $17.12 \pm 5.92$ \\
\hline Targeted behavior difficulties & $17.78 \pm 4.36$ \\
\hline Impulse control difficulties & $18.74 \pm 4.96$ \\
\hline Lack of emotional awareness & $16.21 \pm 4.22$ \\
\hline Limited access to emotional regulation strategies & $24.97 \pm 6.42$ \\
\hline Lack of emotional clarity & $14.33 \pm 4.33$ \\
\hline Reward sensitivity & $8.44 \pm 6.64$ \\
\hline Distress tolerance & $38.83 \pm 9.93$ \\
\hline Impulsivity & $45.61 \pm 18.77$ \\
\hline Aggression & $90.77 \pm 14.89$ \\
\hline
\end{tabular}


Table 3. Statistical assumptions of regression analysis

\begin{tabular}{|c|c|c|c|c|}
\hline \multirow{2}{*}{ Variables } & Collinearity Indicators & \multirow{2}{*}{ Tolerance } & \multirow{2}{*}{ Skewness } & \multirow{2}{*}{ Kurtosis } \\
\hline & Variance Inflation Factor & & & \\
\hline None-acceptance of emotional responses & 1.507 & 0.664 & & \\
\hline Targeted behavior difficulties & 2.470 & 0.405 & & \\
\hline Impulse control difficulties & 2.219 & 0.451 & & \\
\hline Lack of emotional awareness & 1.305 & 0.766 & & \\
\hline Limited access to regulation strategies & 2.040 & 0.490 & & \\
\hline Lack of emotional clarity & 1.506 & 0.664 & & \\
\hline Reward sensitivity & 1.056 & 0.947 & 0.264 & -1.20 \\
\hline Emotional dysregulation & & & -0.412 & -0.31 \\
\hline Distress tolerance & & & 0.629 & 1.18 \\
\hline Impulsivity & & & 0.339 & -0.44 \\
\hline Aggression & & & 0.410 & 0.12 \\
\hline
\end{tabular}

in research variables. MANOVA analysis based on the Lambda effect $(\mathrm{P}=0.213)$ showed that the severity of features did not impact the participants' responses (Table 7).

\section{Discussion}

The current study examined the roles of emotional dysregulation and reward sensitivity in predicting distress tolerance, impulsivity, and aggression among individuals with BPD features.
As predicted, the first result showed among the components of emotional dysregulation, only two components of limited access to emotional regulation strategies and non-acceptance of emotional responses had predictive roles in distress tolerance. Linehan's Biosocial theory supported this result and showed that emotional dysregulation of BPD patients, as an underlying factor, makes these patients use maladaptive coping strategies such as rumination and suppression in the face of distressing emotional states (Linehan, 1993). In line with Carpenter and Trull (2013), emotional dysregulation is an inability to cope with distress and negative affectivity. People with

Table 4. Summary results of multivariate regression analysis for predicting distress tolerance

\begin{tabular}{|c|c|c|c|c|c|c|c|}
\hline Predictor Variable & $\mathbf{R}$ & $\mathbf{R}^{2}$ & $\mathbf{F}$ & Sig. & $\boldsymbol{\beta}$ & $\mathbf{t}$ & Sig. \\
\hline None-acceptance of emotional respons Kurtosis es & & & & & -0.308 & -4.142 & 0.0001 \\
\hline Targeted behavior difficulties & & & & & -0.082 & -0.865 & 0.388 \\
\hline Impulse control difficulties & & & & & -0.135 & -1.498 & 0.136 \\
\hline Lack of emotional awareness & 0.703 & 0.494 & 19.274 & 0.0001 & -0.097 & -1.399 & 0.164 \\
\hline Limited access to emotional regulation strategies & & & & & -0.344 & -3.973 & 0.000 \\
\hline Lack of emotional clarity & & & & & 0.067 & 0.907 & 0.366 \\
\hline Reward sensitivity & & & & & -0.006 & -0.102 & 0.919 \\
\hline
\end{tabular}


Table 5. Summary results of multivariate regression analysis for predicting impulsivity

\begin{tabular}{|c|c|c|c|c|c|c|c|}
\hline Predictor Variables & $\mathbf{R}$ & $\mathbf{R}^{2}$ & $\mathbf{F}$ & Sig. & $\boldsymbol{\beta}$ & $\mathbf{t}$ & Sig. \\
\hline None-acceptance of emotional responses & & & & & 0.011 & 0.277 & 0.782 \\
\hline Targeted behavior difficulties & & & & & 0.012 & 0.230 & 0.818 \\
\hline Impulse control difficulties & & & & & 0.013 & 0.271 & 0.787 \\
\hline Lack of emotional awareness & 0.925 & 0.856 & 117.24 & 0.000 & -0.047 & -1.282 & 0.202 \\
\hline Limited access to emotional regulation strategies & & & & & -0.037 & -0.805 & 0.422 \\
\hline Lack of emotional clarity & & & & & -0.031 & -0.791 & 0.431 \\
\hline Reward sensitivity & & & & & 0.914 & 27.553 & 0.0001 \\
\hline
\end{tabular}

low distress tolerance that have elevated difficulties in emotional regulation are at risk to be engaged in destructive behaviors such as drunkorexia to suppress aversive emotional states (Laghi, Pompili, Bianchi, Lonigro, \& Baiocco, 2020). Accordingly, BPD patients have difficulties tolerating the distress and affective disturbances when following their targets (Carpenter \& Trull, 2013).

Moreover, these patients consider distress unacceptable and intolerable and show hyper-reactivity to stressors (Reilly \& Hines, 2017). These individuals avoid experiencing negative emotionality and subsequently use maladaptive regulatory strategies (non-acceptance of emotional responses) to alleviate distress. Although these strategies can decrease BPD patients' distress in the short term, they may exacerbate negative emotions, increase psychological distress and make one incapable of tolerating distress in the long term.
In this regard, this finding supported prior research (Kuo et al., 2018) and shows that subclinical BPD patients experience emotional distress after suppression conditions, consistent with the current study. Unexpectedly, the results of this study did not support the link between reward sensitivity and distress tolerance. This result is inconsistent with several studies (Simons et al., 2018; Buckner et al., 2007; Allen \& Gabbay, 2013; Bornovalova et al., 2012), stating rewarding behaviors may reduce distress and negative emotions and make one capable of tolerating them in the short term. The difference between clinical samples may explain inconsistent findings. The reported studies in the field of distress tolerance and reward sensitivity have been done on a healthy group, major depressive disorder patients, and substance use disorder. There has not been any research aiming to assess the role of reward sensitivity in negative emotional conditions in the BPD group.

Table 6. Summary results of multivariate regression analysis for predicting aggression

\begin{tabular}{|c|c|c|c|c|c|c|c|}
\hline Predictor Variable & $\mathbf{R}$ & $\mathbf{R}^{2}$ & $\mathbf{F}$ & Sig. & $\beta$ & $\mathbf{t}$ & Sig. \\
\hline None-acce ptance of emotional responses & & & & & -0.011 & -0.111 & 0.912 \\
\hline Targeted behavior difficulties & & & & & 0.012 & -0.341 & 0.734 \\
\hline Impulse control difficulties & & & & & -0.042 & 2.987 & 0.003 \\
\hline Lack of emotional awareness & 0.397 & 0.158 & 3.694 & 0.001 & 0.348 & 0.478 & 0.634 \\
\hline Limited access to emotional regulation strategies & & & & & 0.043 & 1.271 & 0.206 \\
\hline Lack of emotional clarity & & & & & -0.136 & -1.414 & 0.160 \\
\hline Reward sensitivity & & & & & 0.008 & 0.105 & 0.917 \\
\hline
\end{tabular}


Table 7. The multivariate analysis of variance method for analyzing the severity of borderline personality disorder symptoms on the responses of subjects

\begin{tabular}{|c|c|c|c|c|}
\hline Dependent Variables & Effect & Mean Square & $\mathbf{F}$ & Sig. \\
\hline & Lambda & & 1.442 & 0.213 \\
\hline Emotional dysregulation & & 1641.362 & 3.923 & 0.050 \\
\hline Reward sensitivity & & 9.565 & 0.215 & 0.643 \\
\hline Distress tolerance & & 89.298 & 0.904 & 0.343 \\
\hline Impulsivity & & 4.928 & 0.014 & 0.906 \\
\hline Aggression & & 198.597 & 0.894 & 0.346 \\
\hline
\end{tabular}

Additionally, it can be pointed out that the experienced distress in BPD patients is more context-dependent. As suggested in Linehan's theory, these patients show extreme emotional responses to environmental triggers (Linehan, 1993). Hence, their intense negative emotionality cannot reduce only by rewarding actions. Consistent with the theory of emotional waterfalls (Selby et al., 2008), BPD patients experience cognitive and emotional conflict with the situation and extreme values of emotionality when they are exposed to environmental triggers. On the other hand, the second result showed that none of the emotional dysregulation components predicted impulsivity. By contrast, only reward sensitivity had a pivotal role in predicting impulsivity in individuals with BPD symptoms.

As predicted, BPD patients with impulsivity characteristics prefer immediate rewards to delayed ones (Lawrence et al., 2010; Berenson et al., 2016). These patients tend to choose smaller-sooner rewards rather than larger-later options (Marshall \& Kirkpatrick, 2015), suggesting high sensitivity to reward and low sensitivity to loss and punishment in this group. In support of this, instant decision-making in the BPD group may be owing to over-response to positive reinforces. In other words, when these patients are exposed to pleasurable hallmarks in an environment, they encounter processing changes with a bias towards high-risk and high-gain options and eventually conduct high-risk behaviors (Kirkpatrick et al., 2007). Overall, it can be argued that impulsive BPD patients have difficulty resisting rewarding behaviors (Berenson et al., 2020) that lead to behaving unreservedly in an environment that has immediate gains.

The final result of the present study showed that among the components of emotional dysregulation, only impulse control difficulties predict aggression. This result was supported by research (Terzi et al., 2017; Cooper,
Wood, Orcutt, \& Albino 2003) proposed that impulsivity and weakness in impulse control, along with emotional regulation deficits in BPD patients, can cause involvement in destructive and aggressive behaviors. Additionally, impulse control difficulties can mediate the link between boredom and aggression. The experience of boredom may trigger a pressured feeling that leads to impulsivity (Isacescu, Struk, \& Danckert, 2017; Gratz, \& Roemer, 2004), and aggressive behaviors following impulsivity are considered the regulatory strategy to tackle negative emotionality (Cao \& An, 2019; Biolcati, Manchini, \& Trombini, 2018). In line with previous research, lack of inhibitory control may present itself in aggressive behaviors such as risky driving or other destructive actions (Hoptman \& Javitt, 2020; Barati, Pourshahbaz, Nosratabadi, \& Mohammadi, 2020). Moreover, the inability to control impulses serves as a critical factor to aggressive actions against oneself and others under the emotional arousal conditions in BPD patients. Aggressive behaviors such as cutting their bodies, burning themselves with a cigarette, banging their heads as selfdirected aggression, or physical and verbal aggression related to others, are attempts that the BPD patients perform to deal with painful internal feelings.

On the contrary, the results of this study showed no link between reward sensitivity and aggression. This finding was inconsistent with studies that suggest being in an angry condition leads to prefer immediate rewards to delayed ones (Zhao et al., 2017; Bossuyt et al., 2014; Drnas, 2020). The discrepancies in the above studies may be a consequence of the difference between the clinical study groups. Because reported studies have been done on healthy students and children group, and, in contrast, there has not been any research to examine the relationship between reward-seeking and aggression in BPD patients. Besides, the nature of aggression in the 
BPD group may be interpreted as an explanation for the inconsistency of the existing findings. This explanation seems plausible based on Kernberg (2004), who states that BPD patients suffer from excessive aggression. These patients have emotional regulatory deficits and may use verbal or physical aggression to vent their internal anger. The severity of anger and rage in BPD patients leads to destructive behaviors such as self-harm, which aim to vent aversive inner anger and alleviate stress not getting pleasure. Accordingly, it is reasonable to assume that aggressive behaviors may not have a rewarding value by themselves in BPD patients.

\section{Conclusion}

$\mathrm{BPD}$ is a heterogeneous psychiatric disorder, which can have complex causes against diverse diagnostic symptoms. This study found that emotional dysregulation had a remarkable role in distress tolerance and aggression. Besides, impulsivity was only determined by reward sensitivity, that both are proposed as biological mechanisms. Therefore, the present study results can have clinical implications considering both etiology and treatment of BPD patients. Hence, more focus is necessary on psychological interventions based on emotional regulation in treating symptoms related to negative emotionality. Also, the importance of biological treatments such as medication in the field of impulsive and destructive behaviors can be pointed out as practical benefits of the current research.

However, this research has several limitations that need to be considered in future studies. The dropout of participants and lack of cooperation in the second phase of the study are limitations of the present research. Hence, future studies may need a larger sample to examine the variables. Conducting the current study among individuals with BPD features instead of BPD patients can affect the generalizability of the findings to the clinical population. Further investigations should be undertaken in the BPD group. Additionally, this research emphasizes the correlational relationships between variables, which does not allow making causal inferences. Therefore, subsequent studies based on causality can help researchers to understand better the link between reported variables in the context of BPD patients.

\section{Ethical Considerations}

\section{Compliance with ethical guidelines}

All ethical principles were considered in the current research. Written informed consent was obtained from all participants, and they were assured about the confidentiality of their information.

\section{Funding}

This research did not receive any grant from funding agencies in the public, commercial, or non-profit sectors.

\section{Authors' contributions}

All authors equally contributed to preparing this article.

\section{Conflict of interest}

The authors declared no conflict of interest.

\section{Reference}

Allen, J. D., \& Gabbay, F. H. (2013). The amphetamine response moderates the relationship between negative emotionality and alcohol use. Alcoholism: Clinical and experimental research, 37, 348-60. [DOI:10.1111/j.1530-0277.2012.01935.x] [PMID] [PMCID]

American Psychiatric Association. (2013). Diagnostic and Statistical Manual of Mental Disorders (DSM-5). Washington, D.C.: American Psychiatric Publisher. [DOI:10.1176/appi. books.9780890425596]

Azizi, A., Mirzaei, A., \& Shams, J. (2010). Correlation between distress tolerance and emotional regulation with students smoking dependence. Hakim Research Journal, 13(1), 11-18. https://www.sid.ir/en/Journal/ViewPaper.aspx?ID=186015

Baer, M. M., LaCroix, J. M., Browne, J. C., Hassen, H. O., Perera, K. U., \& Soumoff, A., et al. (2018). Impulse control difficulties while distressed: A facet of emotion dysregulation links to non-suicidal self-injury among psychiatric inpatients at military treatment facilities. Psychiatry Research, 269, 419-24. [DOI:10.1016/j.psychres.2018.08.082] [PMID]

Barati, F., Pourshahbaz, A., Nosratabadi, M., \& Mohammadi, Z. (2020). The role of impulsivity, attentional bias and decision-making styles in risky driving behaviors among male drivers in Iran. High Risk Behavioral Addiction, 9(2), e98001. [DOI:10.5812/ijhrba.98001]

Berenson, K. R., Gregory, W. E., Glaser, E., Romirowsky, A Rafaeli, E., \& Yang, X., et al. (2016). Impulsivity, rejection sensitivity, and reactions to stressors in borderline personality disorder. Cognitive Therapy and Research, 40, 510-21. [DOI:10.1007/s10608-015-9752-y] [PMID] [PMCID]

Berenson, K. R., Van De Weert, S. M., Nicolaou, S., Campoverde, C., Rafaeli, E., \& Downey, G. (2020). Reward and punishment sensitivity in borderline and avoidant personality disorders. Journal of Personality Disorders, 34, 475-91. [DOI:10.1521/ pedi_2020_34_475] [PMID]

Bijttebier, P., Beck, I., Claes, L., \& Vandereycken, W. (2009). Grays reinforcement sensitivity theory as a framework for research on personality-psychopathology associations. Clinical Psy- 
chology Review, 29(5), 421-30. [DOI:10.1016/j.cpr.2009.04.002] [PMID]

Biolcati, R., Manchini, G., Trombini, E. (2018). Proneness to boredom and risk behaviors during adolescents free time. Psychological Reports, 121(2),303-23. [DOI:10.1177/0033294117724447] [PMID]

Bornovalova, M. A., Gratz, K. L., Daughters, S. B., Hunt, E. D., \& Lejuez, C. W. (2012). Initial RCT of a distress tolerance treatment for individuals with substance use disorders. Drug and Alcohol Dependence, 122(1-2), 70-6. [DOI:10.1016/j.drugalcdep.2011.09.012] [PMID] [PMCID]

Bornovalova, M. A., Matusiewicz, A., \& Rojas, E. (2011). Distress tolerance moderates the relationship between negative affect intensity with borderline personality disorder levels. Comprehensive Psychiatry, 52(6), 744-53. [DOI:10.1016/j. comppsych.2010.11.005] [PMID] [PMCID]

Bossuyt, E., Moors, A., \& De Houwer, J. (2014). On angry approach and fearful avoidance: The goal-dependent nature of emotional approach and avoidance tendencies. Journal of Experimental Social Psychology, 50, 118-24. [DOI:10.1016/j. jesp.2013.09.009]

Buckner, J. D., Keough, M. E., \& Schmidt, N. B. (2007). Problematic alcohol and cannabis use among young adults: The roles of depression and discomfort and distress tolerance. Addictive Behaviors, 32(9), 1957-63. [DOI:10.1016/j.addbeh.2006.12.019] [PMID] [PMCID]

Buss, A. H., \& Perry, M. (1992). The aggression questionnaire. Journal of Personality and Social Psychology, 63(3), 452. [DOI:10.1037/0022-3514.63.3.452] [PMID]

Cao, Q., \& An, J. (2020). Boredom proneness and aggression among people with substance use disorder: The mediating role of trait anger and impulsivity. Journal of Drug Issues, 50(1), 77-88. [DOI:10.1177/0022042619886822]

Carpenter, R. W., \& Trull, T. J. (2013). Components of emotion dysregulation in borderline personality disorder: A review. Current Psychiatry Reports, 15(1), 1-8. [DOI:10.1007/s11920012-0335-2] [PMID] [PMCID]

Chapman, A. L., Leung, D. W., \& Lynch, T. R. (2008). Impulsivity and emotion dysregulation in borderline personality disorder. Journal of Personality Disorders, 22(2), 148-64 [DOI:10.1521/pedi.2008.22.2.148] [PMID]

Cooper, M. L., Wood, P. K., Orcutt, H. K., \& Albino, A. (2003) Personality and the predisposition to engage in risky or problem behaviors during adolescence. Journal of Personality and Social Psychology, 84(2), 390-410. [DOI:10.1037/00223514.84.2.390] [PMID]

Curry, I., Luk, J. W., Trim, R. S., Hopfer, C. J., Hewitt, J. K., \& Stallings, M. C., et al. (2018). Impulsivity dimensions and risky sex behaviors in an at-risk young adult sample. Archives of Sexual Behavior, 47(2), 529-36. [DOI:10.1007/s10508-017-1054-x] [PMID] [PMCID]

Daughters, S. B., Lejuez, C. W., Kahler, C. W., Strong, D. R., \& Brown, R. A. (2005). Psychological distress tolerance and duration of most recent abstinence attempt among residential treatment-seeking substance abusers. Psychology of Addictive Behaviors, 19(2), 208-11. [DOI:10.1037/0893-164X.19.2.208] [PMID]

Dawe, S., Gullo, M. J., \& Loxton, N. J. (2004). Reward drive and rash impulsiveness as dimensions of impulsivity: Implica- tions for substance misuse. Addictive Behaviors, 29(7), 1389-405. [DOI:10.1016/j.addbeh.2004.06.004] [PMID]

Dixon-Gordon, K. L., Turner, B. J., Rosenthal, M. Z., \& Chapman, A. L. (2017). Emotion regulation in borderline personality disorder: An experimental investigation of the effects of instructed acceptance and suppression. Behavior Therapy, 48(6), 750-64. [DOI:10.1016/j.beth.2017.03.001] [PMID]

Drnas, M. S. (2020). The role of gender in the relationship between reinforcement sensitivity and aggression among adolescents. International of Emotional Education, 12(1), 3-18. https://eric. ed.gov/?id=EJ1251782

Ebner-Priemer, U., Kuo, J., Schlotz, W., Kleindienst, N., Rosenthal, M. Z., \& Detterer, L., et al. (2008). Distress and affect regulation in patients with Borderline Personality Disorder: A psychophysiological ambulatory monitoring study. Journal of Nervous and Mental Disease, 196(4), 314-20. [DOI:10.1097/NMD.0b013e31816a493f] [PMID]

Esmailian, N., Dehghani, M., Koster, E., \& Hoorelbeke, K. (2019). Early maladaptive schemas and borderline personality disorder features in a nonclinical sample: A network analysis. Clinical Psychology \& Psychotherapy, 26(3), 388-98. [DOI:10.1002/cpp.2360] [PMID]

Evans, D., Howard, M., Dudas, R., Denman, C., \& Dunn, B. D. (2013). Emotion regulation in borderline personality disorder: Examining the consequences of spontaneous and instructed use of emotion suppression and emotion acceptance when viewing negative films. Journal of Experimental Psychopathology, 4(5), 54665. [DOI:10.5127/jep.029512]

Evenden, J. L. (1999). Varieties of impulsivity. Psychopharmacology, 146(4), 348-61. [DOI:10.1007/PL00005481] [PMID]

Germain, C. L., \& Kangas, M. (2015). Trait anger symptoms and emotion regulation: The effectiveness of reappraisal, acceptance and suppression strategies in regulating anger. Behaviour Change, 32(1), 35-45. [DOI:10.1017/bec.2014.28]

Goodman, M., \& New, A. (2000). Impulsive aggression in borderline personality disorder. Current Psychiatry Reports, 2(1), 56-61. [DOI:10.1007/s11920-000-0043-1] [PMID]

Gratz, K. L., \& Roemer, L. (2004). Multidimensional assessment of emotion regulation and dysregulation: Development, factor structure, and initial validation of the difficulties in emotion regulation scale. Journal of Psychopathology and Behavioral Assessment, 26(1), 41-54. [DOI:10.1023/B:JOBA.0000007455.08539.94]

Hoptman, M., \& Javitt, D. (2020). A mediation reanalysis of pathways from frontal dysfunction to aggression: The role of emotional impulsivity. Biological Psychiatry, 87(9), S356. [DOI:10.1016/j. biopsych.2020.02.913]

Isacescu, J., Struk, A. A., Danckert, J. (2017). Cognitive and affective predictors of boredom proneness. Cognition and Emotion 31(8), 1741-8. [DOI:10.1080/02699931.2016.1259995] [PMID]

Jakubczyk, A., Trucco, E. M., Kopera, M., Kobyliński, P., Suszek, H., \& Fudalej, S., et al. (2018). The association between impulsivity, emotion regulation, and symptoms of alcohol use disorder. Journal of Substance Abuse Treatment, 91, 49-56. [DOI:10.1016/j.jsat.2018.05.004] [PMID] [PMCID]

Kakabraei, K., \& Azami, E. (2017). [Predicting distress tolerance based-on behavioural brain systems and emotional dysregulation (Persian)]. Journal of Tandorosti Nasim. [Online Publishing]. http://jfh.iausari.ac.ir/article_653745.html 
Kernberg, O. (2004). Aggressivity, narcissism, and self-destructiveness in the psychotherapeutic relationship. New Haven \& Nondon: Yle University Press. [DOI:10.12987/ yale/9780300101805.001.0001]

Kirkpatrick, T., Joyce, E., Milton, J., Duggan, C., Tyrer, P., \& Rogers, R. D. (2007). Altered emotional decision-making in prisoners with borderline personality disorder. Journal of Personality Disorders, 21(3), 243-61. [DOI:10.1521/pedi.2007.21.3.243] [PMID]

Krause-Utz, A., CackowskI, S., Daffner, S., Sobanski, E., Plichta, M. M., \& Bohus, M, et al. (2016). Delay discounting and response inhibition under acute experimental stress in women with borderline personality disorder and adult attention deficit hyper activity disorder. Psychological Medicine, 46(15), 3137. [DOI:10.1017/S0033291716001677] [PMID]

Kuo, J. R., Fitzpatrick, S., Krantz, L. H., \& Zeifman, R. J. (2018). How do you choose and how well does it work?: The selection and effectiveness of emotion regulation strategies and their relationship with borderline personality disorder feature severity. Cognition and Emotion, 32(3), 632-40. [DOI:10.108 0/02699931.2017.1330254] [PMID]

Laghi, F., Pompili, S., Bianchi, D., Lonigro, A., \& Baiocco, R. (2020). Exploring the association between psychological distress and drunkorexia behaviors in non-clinical adolescents: The moderating role of emotional dysregulation. Eating and Weight Disorders-Studies on Anorexia, Bulimia and Obesity, 1-10. [DOI:10.1007/s40519-020-00912-9] [PMID]

Lawrence, K. A., Allen, J. S., \& Chanen, A. M. (2010). Impulsivity in borderline personality disorder: Reward-based decision-making and its relationship to emotional distress. Journal of Personality Disorders, 24(6), 785-99. [DOI:10.1521/ pedi.2010.24.6.785] [PMID]

Lejuez, C. W., Read, J. P., Kahler, C. W., Richards, J. B., Ramsey, S. E., \& Stuart, G. L, et al. (2002). Evaluation of a behavioral measure of risk taking: the Balloon Analogue Risk Task (BART). Journal of Experimental Psychology: Applied, 8(2), 75. [DOI:10.1037/1076-898X.8.2.75] [PMID]

Leyro, T. M., Zvolensky, M. J., \& Bernstein, A. (2010). Distress tolerance and psychopathological symptoms and disorders: A review of the empirical literature among adults. Psychological Bulletin, 136(4), 576-600. [DOI:10.1037/a0019712] [PMID] [PMCID]

Linehan, M. M. (1993). Dialectical behavior therapy for treatment of borderline personality disorder: Implications for the treatment of substance abuse. NIDA Research Monograph, 137, 201. [DOI:10.1037/e495912006-012]

Marshall, A. T., Kirkpatrick, K. (2015). Mechanisms of impulse choice: The role of reward processes. Behavioral Processes, 1-15. https:/ / www.researchgate.net/publication/284443118_ Mechanisms_of_impulsive_choice_III_The_role_of_reward_ processes

Martino, F., Caselli, G., Berardi, D., Fiore, F., Marino, E., \& Menchetti, M., et al. (2015). Anger rumination and aggressive behaviour in borderline personality disorder. Personality and Mental Health, 9(4), 277-87. [DOI:10.1002/pmh.1310] [PMID]

Morey, L. (1991). Professional manual for the personality assessment inventory. Odessa, FL: Psychological Assessment Resources. [DOI:10.1037/t03903-000]
Muratori, P., Pisano, S., Milone, A., \& Masi, G. (2017). Is emotional dysregulation a risk indicator for auto-aggression behaviors in adolescents with oppositional defiant disorder? Journal of Affective Disorders, 208, 110-2. [DOI:10.1016/j. jad.2016.08.052] [PMID]

Nasri, S., Nazari, M. A., Mighani, E., Shahrokhi, H., \& Goudarzi, I. (2018). [The intraction between risk-taking and behavioural inhibition in ADHD with and without conduct disorder in comparison to normal children (Persian)]. Psychology and Cognition Psychiatry, 5(4), 63-77. [DOI:10.29252/shenakht.5.4.63]

Paret, C., Jennen-Steinmetz, C., \& Schmahl, C. (2016). Disadvantageous decision making in borderline personality disorder: partial support from a meta-analytic review. Neuroscience and Biobehavioral Reviews, 72, 301-9. [DOI:10.1016/j.neubiorev.2016.11.019] [PMID]

Paris, J., Gunderson, J., \& Weinberg, I. (2007). The interface between borderline personality disorder and bipolar spectrum disorders. Comprehensive Psychiatry, 48(2), 145-54. [DOI:10.1016/j.comppsych.2006.10.001] [PMID]

Reilly, M. E., \& Hines, D. A. (2017). Distress tolerance as a mediator of the association between Borderline Personality Symptoms and Obsessive Relational Intrusion: An exploratory analysis. Journal of Interpersonal Violence, 35(19-20), 3833-48. [DOI:10.1177/0886260517712274] [PMID]

Samani, S. (2008). [Study of reliability and validity of the Buss and Perry's aggression questionnaire (Persian)]. Iranian Journal of Psychiatry and Clinical Psychology, 13(4), 359-65. http:// ijpcp.iums.ac.ir/article-1-356-en.html

Sansone, R. A., \& Sansone, L. A. (2012). Borderline personality and externalized aggression. Innovations in Clinical Neuroscience, 9(3), 23-6. [PMCID] [PMID]

Scott, L, N., Stepp, S. D., \& Pilkonis, P. A. (2014). Prospective associations between features of borderline personality disorder, emotion dysregulation, and aggression. Personal Disorders, 5(3), 278-88. [DOI:10.1037/per0000070] [PMID] [PMCID]

Sebastian, A., Jung, P., Krause-Utz, A., Lieb, K., Schmahl, C., \& Tüscher, O. (2014). Frontal dysfunctions of impulse controla systematic review in borderline personality disorder and attention-deficit/hyperactivity disorder. Frontiers in Human Neuroscience, 8, 698. [DOI:10.3389/fnhum.2014.00698] [PMID] [PMCID]

Selby, E. A., Anestis, M. D., \& Joiner, T. E. (2008). Understanding the relationship between emotional and behavioral dysregulation: Emotional cascades. Behaviour Research and Therapy 46(5), 593-611. [DOI:10.1016/j.brat.2008.02.002] [PMID]

Simons, J. S., \& Gaher, R. M. (2005). The Distress Tolerance Scale: Development and validation of a self-report measure. Motivation and Emotion, 29(2), 83-102. [DOI:10.1007/s11031-005-7955-3]

Simons, R. M., Sistad, R. E., Simons, J. S., \& Hansen, J. (2018). The role of distress tolerance in the relationship between cognitive schemas and alcohol problems among college students Addictive behaviors, 78, 1-8. [DOI:10.1016/j.addbeh.2017.10.020] [PMID] [PMCID]

Terzi, L., Martino, F., Berardi, D., Bortolotti, B., Sasdelli, A., \& Menchetti, M. (2017). Aggressive behavior and self-harm in Borderline Personality Disorder: The role of impulsivity and emotion dysregulation in a sample of outpatients. Psychiatry Research, 249, 321-6. [DOI:10.1016/j.psychres.2017.01.011] [PMID] 
Trull, T. J. (1995). Borderline personality disorder features in nonclinical young adults: Identification and validation. Psychological Assessment, 7, 33-41. [DOI:10.1037/1040-3590.7.1.33]

Trull, T. J., Jahng, S., Tomko, R. L., Wood, P. K., \& Sher, K. J. (2010). Revised NESARC personality disorder diagnoses: gender, prevalence, and comorbidity with substance dependence disorders. Journal of Personality Disorders, 24(4), 412-26. [DOI:10.1521/pedi.2010.24.4.412] [PMID] [PMCID]

Volker, K. A., Spitzer, C., Limberg, A., Grabe, H. J., Freyberger, H. J., \& Barnow, S. (2009). Exekutive dysfunktionen bei patientinnen mit borderline-persönlichkeitsstörung unter berücksichtigung von impulsivität und depressivität. Psychotherapie Psychosomatik Medizinische, 59(07), 264-72. [DOI:10.1055/s-2008-1067437] [PMID]

Vollum, B., Richardson, P., McKie, S., Elliot, R., Dolan, M., \& Deakin, B. (2007). Neuronal correlates of reward and loss in cluster B personality disorders: A functional magnetic resonance imaging study. Psychiatry Research: Neuroimaging 156(2), 151-67. [DOI:10.1016/j.pscychresns.2007.04.008] [PMID]

Zhao, J., Kirwen, N., Johnson, J., \& Vigo, R. (2017). Anger and intertemporal choice: The behavioral approach system and the interactive effects of trait and state anger. Personality and Individual Differences, 110, 60-64. [DOI:10.1016/j.paid.2017.01.022] 
This Page Intentionally Left Blank 